\title{
NONREGULAR EXTREME POINTS IN THE SET OF MINKOWSKI ADDITIVE SELECTIONS
}

\author{
KRZYSZTOF PRZESŁAWSKI
}

(Communicated by William J. Davis)

\begin{abstract}
A function $s: \mathscr{K}^{n} \rightarrow \mathbb{R}^{n}$, defined on the family $\mathscr{K}^{n}$ of all compact convex and nonempty sets in $\mathbb{R}^{n}$, is called a Minkowski additive selection, provided $s(A+B)=s(A)+s(B)$ and $s(A) \in A$, whenever $A, B \in \mathscr{K}^{n}$. We confirm the conjecture [6] that there exist extremal selections which are not regular $\left(s\right.$ is regular if $\left.s(A) \in \operatorname{ext} A, A \in \mathscr{K}^{n}\right)$.
\end{abstract}

Let $\mathscr{K}^{n}$ denote the family of all convex compact nonempty subsets of $\mathbb{R}^{n}$. A mapping $T: \mathscr{K}^{n} \rightarrow \mathbb{R}^{n}$ is called Minkowski additive, or simply additive, if $T(A+B)=T(A)+T(B)$ for all $A, B \in \mathscr{K}^{n}$. Let $\mathscr{L}^{n}$ be the vector space of all additive mappings equipped with the weakest topology under which all evaluations $\mathscr{L}^{n} \ni T \rightarrow T(A), A \in \mathscr{K}^{n}$, are continuous. It can be easily seen that the set $\mathscr{S}^{n} \subset \mathscr{L}^{n}$ of all selections, i.e., the mappings having the property $T(A) \in A$, is convex and compact. Let $\mathscr{E}^{n}$ be the set of all extreme points of $\mathscr{S}^{n}$. An element $s \in \mathscr{S}^{n}$ is called regular if $s(A) \in \operatorname{ext} A$, where ext $A$ denotes the set of all extremal points of $A$. The set of all regular selections will be denoted by $\mathscr{R}^{n}$. Obviously, if $s \in \mathscr{R}^{n}$, then $s \in \mathscr{E}^{n}$.

Živaljević [6] conjectured that there exist nonregular extremal points in $\mathscr{S}^{n}$. The following result confirms this supposition.

Theorem. For every $n \geq 2$, there exists a closed face of $\mathscr{S}^{n}$ disjoint with $\mathscr{R}^{n}$.

To prove the theorem, we shall need some additional notions and definitions. Let us define the support function $h(A, x)$ of $A \in \mathscr{K}^{n}$ at $x$ as follows:

$$
h(A, x)=\sup \{\langle a, x\rangle: a \in A\},
$$

where $\langle\cdot, \cdot\rangle$ denotes the scalar product. By $\Omega_{k}$ we denote the Stiefel manifold of $k$-frames; that is, the ordered $k$-tuples $\omega=\left(x_{1}, \ldots, x_{k}\right)$ of orthonormal vectors in $\mathbb{R}^{n}$. For $\omega \in \Omega_{k}$, we define the $\omega$-face $V_{\omega}(A)$ of $A$ inductively: Let $V_{x_{1}}(A)=\left\{a \in A:\left\langle a, x_{1}\right\rangle=h\left(A, x_{1}\right)\right\}$ and suppose that we have already defined $V_{\omega^{\prime}}$ for $\omega^{\prime}=\left(x_{1}, \ldots, x_{k-1}\right)$. Then $V_{\omega}(A)=V_{x_{k}}\left(V_{\omega^{\prime}}(A)\right)$. Subsequently, for $V_{\omega}(A)$, we define its position vector $H_{\omega}(A)$ as follows:

$$
H_{x_{1}}(A)=h\left(A, x_{1}\right) x_{1}, \quad H_{\omega}(A)=H_{\omega^{\prime}}(A)+h\left(V_{\omega^{\prime}}(A), x_{k}\right) x_{k} .
$$

Received by the editors December 1, 1991.

1991 Mathematics Subject Classification. Primary 52A20; Secondary 52A07.

Key words and phrases. Selections, convex sets, extremal points. 
It was the main result of [6] that $\mathscr{R}^{n}=\left\{H_{\omega}: \omega \in \Omega_{n}\right\}$. Basic properties of the face mappings can be found in [2,5].

Suppose now that $A \in \mathscr{K}^{2}$. Let us denote by $s_{0}(A)$ the center of the smallest rectangle containing $A$, which has its sides parallel to the coordinate axes. It is easy to observe that $s_{0}(A) \in A$. Moreover, $s_{0}(A)$ can be expressed by the formula

$$
\begin{aligned}
s_{0}(A)= & \frac{1}{2}\left(h\left(A, e_{1}\right)-h\left(A,-e_{1}\right)\right) e_{1} \\
& +\frac{1}{2}\left(h\left(A, e_{2}\right)-h\left(A,-e_{2}\right)\right) e_{2}
\end{aligned}
$$

where $e_{1}, e_{2}$ denote vectors of the standard basis in $\mathbb{R}^{2}$. Obviously, $s_{0}$ is an additive selection on $\mathscr{K}^{2}$. This selection has already been mentioned in $[1,4]$. Proposition. The minimal closed face of $\mathscr{S}^{2}$ containing $s_{0}$ is disjoint with $\mathscr{R}^{2}$. Proof. For every pair $\varepsilon_{1}, \varepsilon_{2} \in\{-1,1\}$, we define the triangle $T\left(\varepsilon_{1}, \varepsilon_{2}\right)=$ $\operatorname{conv}\left\{0, \varepsilon_{1} e_{1}, \varepsilon_{2} e_{2}\right\}$. It is clear that $s_{0}\left(T\left(\varepsilon_{1}, \varepsilon_{2}\right)\right)=\left(\varepsilon_{1} e_{1}+\varepsilon_{2} e_{2}\right) / 2$. Hence for every selection $s$ belonging to the minimal closed face containing $s_{0}$ we have

$$
s\left(T\left(\varepsilon_{1}, \varepsilon_{2}\right)\right) \in\left[\varepsilon_{1} e_{1}, \varepsilon_{2} e_{2}\right] .
$$

On the other hand, it can be easily seen that no regular point $H_{\omega}, \omega \in \Omega_{2}$, can satisfy all the relations $(*)$ resulting when $\varepsilon_{1}$ and $\varepsilon_{2}$ run over $\{-1,1\}$.

Proof of the theorem. Let us regard $\mathbb{R}^{2}$ as embedded into $\mathbb{R}^{n}$ in an obvious manner. Let $\omega \in \Omega_{n-2}$ consist of elements orthogonal to $\mathbb{R}^{2}$. It is clear that $V_{\omega}(A)-H_{\omega}(A) \in \mathbb{R}^{2}$ for every $A \in \mathscr{K}^{n}$. Consequently, the following mapping is well defined:

$$
s_{\omega}(A)=s_{0}\left(V_{\omega}(A)-H_{\omega}(A)\right)+H_{\omega}(A) .
$$

It is an easy exercise to prove that $s_{\omega}$ is an additive selection on $\mathscr{K}^{n}$. Furthermore, it follows from the proposition that the minimal face containing $s_{\omega}$ is disjoint with $\mathscr{R}^{n}$.

Question. Is it true that $s_{\omega} \in \mathscr{E}^{n}$ ?

For further information on the topic of extremal selections the reader is referred to the more extensive study [3].

\section{REFERENCES}

1. Z. Artstein, Stabilizing selections of differential inclusions, Weizmann Institute of Science. preprint.

2. Cz. Olech, A note concerning extremal points of a convex set, Bull. Acad. Sci. Ser. Sci. Math. Fis. Nat. 13 (1965), 347-352.

3. K. Przeslawski, Faces of convex sets and Minkowski additive selections, unpublished.

4. K. Przesławski and D. Yost, Continuity properties of selectors and Michael's Theorem. Michigan Math. J. 36 (1989), 113-134.

5. G. Stefani and P. Zecca, Multivalued differential equations on manifolds with application to control theory, Illinois Math. J. 24 (1980), 560-575.

6. R. Živaljević, Extremal Minkowski additive selections of compact convex sets, Proc. Amer. Math. Soc. 105 (1989), 697-700.

Instytut Matematyki, Wyższa Szkota Inżynierska, Ul. Podgórna 50, 65-246 Zielona Góra, Poland 